\title{
Classification of Adolescent Suicide Based on Student Suicide Reports
}

\author{
Hoin Kwon', Hyun Ju Hong ${ }^{2,3}$, and Yong-Sil Kweon ${ }^{2,4}$ \\ ${ }^{1}$ Department of Counseling Psychology, Jeonju University, Jeonju, Korea \\ ${ }^{2}$ Suicide and School Mental Health Institute, Hallym University, Chuncheon, Korea \\ ${ }^{3}$ Department of Psychiatry, Hallym University Sacred Hospital, Hallym University, Anyang, Korea \\ ${ }^{4}$ Department of Psychiatry, The Catholic University of Korea College of Medicine, Seoul, Korea
}

\begin{abstract}
Exploring the risk factors of adolescent suicide is important for effective suicide prevention. This study explored the clustering of adolescent suicides based on six risk factors: mental disorder, broken family, depression, anxiety, previous suicide attempts, and deviant behaviors. Using 173 student suicide reports obtained from the Ministry of Education, we evaluated the associations between suicide and variables related to mental disorders; dysfunctional family life; depression and anxiety; previous suicide attempts; deviant behaviors such as drinking and smoking; and school life characteristics, including attendance and discipline, problems within the past year, and incidents prior to suicide. In addition, reports of warning signs just before suicide were included in the analysis. The two-stage cluster analysis classified the students into three clusters: the silent type (cluster 1; 48.55\%), in which no risk factors were observed; environmental-risk type (cluster 2: 24.28\%), which featured a high frequency of broken households, deviant behaviors such as smoking/drinking and running away from home; and depressive type (cluster 3:27.17\%), which featured a high frequency of mental health problems such as depression, anxiety, and suicide attempts. Identifying the sub-types of adolescent suicide may help to inform tailored suicide prevention and intervention strategies in school.
\end{abstract}

Key Words: Adolescent; Suicide; Risk factors; Cluster analysis.

Received: August 3, 2020 / Revision: September 1, 2020 / Accepted: September 3, 2020

Address for correspondence: Yong-Sil Kweon, Department of Psychiatry, The Catholic University of Korea College of Medicine, 222 Banpo-daero, Seocho-gu, Seoul 06591, Korea

Tel: +82-31-820-3055, Fax: +82-31-847-3630, E-mail: yskwn@catholic.ac.kr

\section{INTRODUCTION}

Suicide ranks third in the list of the top 10 causes of death among adolescents worldwide [1] and first among the top 10 causes of death among adolescents in South Korea since 2009 [2]. The consequences of suicide to the community, including the family and relatives of the individual who died, further underscores the need to curtail the frequency of suicide.

Adolescent suicide is the result of complex interactions between individuals, families, and their communities, and many studies have identified mental disorder as a major risk factor for adolescent suicide [3]. Findings implicating the dissolution of a family due to the separation of or divorce of the parents in suicide have been consistent [4-6]. Further, suicide attempts [7], depression and anxiety [8], and behavioral problems [9] have been identified as other risk factors for adolescent suicide as well. However, as not every adolescent who commits suicide follows the same behavioral pattern, the various

This is an Open Access article distributed under the terms of the Creative Commons Attribution Non-Commercial License (https://creativecommons.org/licenses/by-nc/4.0) which permits unrestricted non-commercial use, distribution, and reproduction in any medium, provided the original work is properly cited. trajectories that induce the suicide of adolescents with these risk factors must be explored in depth. Psychological autopsy has been introduced as an important part of this effort. Psychological autopsy is the process of reviewing the state of the deceased for a certain time period before his or her suicide by assessing the records left by and the statements of people around the deceased individuals to speculate the cause of suicide; it has become an essential research tool by which to understand the complex causes of suicide [10].

Schools are places where most adolescents spend most of their time. According to research, signs indicating a heightened risk of suicide more frequently present at school than at home, and students are more likely to gain protective factors against suicide at school: e.g., by bonding with someone at school who can help with the suicide problem [11]. This finding suggests that schools can play a critical role in suicide prevention and intervention. Nevertheless, studies have rarely conducted an in-depth analysis of suicide within the school system [12].

Beginning in 2015, the Ministry of Education has obligated all elementary, middle and high schools to submit a detailed 
and comprehensive annual "Student Suicide Report" to the Office of Education and Ministry of Education. The content provided in these reports has populated the student suicide database [13].

This study aims to analyze the features of suicide among Korean students based on the 2018 and 2019 data reported in the student suicide reports. In particular, we aim to examine how student suicide can be classified via cluster analysis based on the six previously identified risk factors for adolescent suicide: mental disorder, previous suicide attempts, depression, anxiety, family breakup, and deviant behaviors. Further, by exploring the differences in the changes or stressful situations immediately before suicide, as well as verbal and behavioral signs before suicide, between the clustered groups, we attempt to speculate the process that leads to suicide among adolescents and gain conclusions that may help schools to develop suicide prevention and intervention strategies

\section{METHODS}

\section{Participants}

This study used data from student suicide reports submitted to the Suicide and School Mental Health Institute in 2018 and 2019 [14,15]. Two teachers, including the homeroom teacher, wrote the suicide report. A total of 258 cases of student suicides were reported from January 2017 to December 2018. After excluding 75 cases due to their missing data required for cluster analysis (i.e., namely status of mental disorder, family breakup, suicide attempts, deviant behaviors, depression, and anxiety), the data from the remaining 173 cases were included in the analysis.

\section{Instruments}

\section{Demographic factors}

The base questionnaire included the students' demographic factors, including sex, age, and school level. Further, the student's family's socioeconomic status, as reported by the teachers, was assessed as high/middle/low. Families in which both parents did not reside together due to divorce, bereavement, or separation were defined as disorganized families. Finally, intimacy with parents was rated on a 6-point scale from very weak to very strong. A higher score indicated greater intimacy.

\section{Strengths and Difficulties Questionnaire}

In this study, the children's and adolescents' mental health problems were screened using the Strengths and Difficulties Questionnaire developed by Goodman and standardized into Korean by Ahn et al. [16]. The strengths are assessed by the questionnaire through five items for social orientation, and difficulties through 25 items: five for each of the subscales of hyperactivity, emotional symptoms, behavioral problems, and peer-relationship problems. The internal consistency (Cronbach's $\alpha$ ) computed in this study was 0.81 for strengths and 0.80 for difficulties.

\section{Deviant behaviors}

In this study, a single item that asked about various deviant behaviors in the Student Suicide Report was used: specifically, about smoking, drinking, running away from home, theft, school violence perpetration, and others. The presence of any one of these items was analyzed.

\section{Self-destructive behaviors and psychiatric problems}

In the Student Suicide Report, two items asking about nonsuicidal self-injury and suicide attempts in the past year and the presence of mental disorder and its diagnosis were included. Further, in terms of students' emotional and behavioral states in the past two months, the presence of depression or anxiety was analyzed.

\section{School life}

Students' grades were classified as good, moderate, or poor. Whether the student had good attendance without unauthorized absences or tardiness was also considered. School belongingness was assessed on a 4-point scale from 1 to 4, where higher scores indicates a greater sense of belonging. Relationships with teachers were also rated on a 4-point scale from 1 to 4 , where higher scores indicate better relationships. Further, whether the school-based mental health screening test was performed and whether the students were classified as normal or in need of management were analyzed.

\section{Problems in the past year and events immediately before suicide}

Problems within the past year were categorized into five types of problems: personal, addiction, family, friends, and school study. Personal problems include dissatisfaction with looks, physical health problems, and mental health problems. Addiction problems include addictions to gaming, the internet, smartphone, pornography, and drugs. Family problems include domestic violence, financial difficulties, physical and mental health problems of family members, parent-child conflict, and conflict between parents. Friend problems include extortion, violence victimization and perpetration, bullying/ cyberbullying victimization and perpetration, joining of school gang, dating, and intimate relationship problems. School study problems include pessimism about poor grades and dropping grades, burden with high competition in school and learning 
workload, burden of study and prospective career focus, fear of academic failure, and parental pressure concerning grades. Having at least one problem in each of the five subscales was included in the analysis.

Events immediately preceding suicide include conflict with parents, conflict with friends, dating and relationship problems, conflict with siblings, career- and grade-related events, punishment, and others. The presence of each event was included.

\section{Warning signs before death}

Students' warning signs before death consist of three types: verbal, behavioral, and emotional. Eight verbal signs, including "talking about suicide" and "ask about how to commit suicide," were considered. Behavioral signs contained 12 items, including "changes in sleep" and "handle personal matters." Three emotional signs, such as "changes in emotional state" and "avoid social contact," were included. After rating these three types independently, the frequency of the items within each cluster type was computed, and the total scores for the three types of signs (verbal, behavioral, and emotional) were summed.

\section{Study procedure}

This study was approved by the Institutional Review Board of the Hallym University School of Medicine (2016-I044). First, the participants' demographic and clinical features were analyzed using descriptive statistics. To examine how the adolescents' risk factors could be classified, a two-step cluster analysis was performed that considered psychiatric disorders, family breakup, past suicide attempts, depression, and anxiety. The differences in demographic features, clinical and suicide-related features, and school and behaviors between the groups were analyzed with a cross tabulation analysis and oneway analysis of variance (ANOVA) followed by the Scheffe post-hoc test. All statistical analyses were performed using SPSS 25.0 (IBM Corp., Armonk, NY, USA).

\section{RESULTS}

\section{Participants' demographic features}

Our analysis included the data provided by 173 student suicide reports. The mean age of the students was 16.05 (standard deviation=1.79), and $56.1 \%$ of the sample was comprised of male students. A majority of the students were attending high school (64.7\%), followed by middle school (32.4\%) and elementary school (2.9\%). The most common method of suicide was jumping $(71.1 \%)$, followed by hanging $(24.9 \%)$, and gas poisoning (2.3\%). Family breakups, past suicide attempts, psychiatric diagnosis, depression, anxiety, and deviant behaviors were analyzed using frequency analysis. Of the participants,
$19.1 \%$ were diagnosed with a psychiatric disorder, $23.7 \%$ were in a broken family, $12.7 \%$ had deviant behaviors, $5.8 \%$ had engaged in previous suicide attempts, $26.6 \%$ had depression, and $11.6 \%$ had anxiety. The most common psychiatric diagnosis among the 33 students with a psychiatric condition was depression (57.6\%), followed by unknown or unclear diagnosis (24.2\%); schizophrenia (6.1\%); and finally attention-defi$\mathrm{cit} /$ hyperactivity disorder, panic disorder, obsessive-compulsive disorder, and conversion disorder (3.0\% each).

\section{Clustering results}

A two-step cluster analysis was performed to analyze the types of suicide among adolescents. Based on previous studies, the six risk factors of adolescent suicide, namely family breakup, past suicide attempts, mental disorders, depression, anxiety, and deviant behaviors were included in the cluster analysis. First, the most appropriate number of clusters without hurting cluster quality was determined by considering the Akaike information criterion (AIC), Bayesian information criterion (BIC), and distance measures. This calculation helped to inform the hierarchical cluster analysis based on this was performed. With respect to AIC, BIC, and distance measures, three clusters were determined to be the most appropriate, and cluster quality was good at a silhouette measure of cohesion and separation of 0.7 [17]. The variable importance was the highest for depression, followed by family breakup, mental disorder, anxiety, deviant behaviors, and past suicide attempts.

Cluster 1 included students with no clear presentation of any risk factors (depression $0.0 \%$, family breakup $0.0 \%$, mental disorder $0.0 \%$, anxiety $0.0 \%$, deviant behaviors $0.0 \%$, previous suicide attempts $0.0 \%$ ); this group was named the silent type. Cluster 2 had higher frequencies of family breakup and deviant behaviors than did the other groups; this group was named the environmental-risk type (depression, 16.7\%; family breakup, $81.0 \%$; mental disorder, $2.4 \%$; anxiety, $0.0 \%$; deviant behaviors, $38.1 \%$; previous suicide attempt, $0.0 \%$ ). Cluster 3 had higher prevalences of depression, mental disorders, and previous suicide attempts relative to the other groups (depression, $83.0 \%$; family breakup, $14.9 \%$; mental disorder, $68.1 \%$; anxiety, $42.6 \%$; deviant behaviors, $12.8 \%$; previous suicide attempts, $21.3 \%)$; this group was named the depressive type.

\section{Demographics of three clusters}

The differences in the demographic data between the three clusters were analyzed using one-way ANOVA and Pearson's chi-squared test. There were no significant differences in age between the three clusters. Regarding sex ratio, clusters 1 and 2 had a higher percentage of males, while cluster 3 had a higher percentage of females; none of the differences were statistically significant. The analysis of residual structures using 
Pearson residuals revealed that the three clusters were significantly different in terms of socioeconomic status: cluster 1 had a significantly lower percentage of students with a "low" socioeconomic status than did the other groups, while cluster 2 had a significantly higher percentage of students rated to have a "low" socioeconomic status. Table 1 shows the demographic characteristics of these groups.

\section{Strengths and difficulties of three clusters}

The differences in strengths and difficulties among the three clusters were analyzed using one-way ANOVA. There were no significant differences in the social orientation (strength), hyperactivity, or behavioral problems subscales (difficulties) between the three clusters. On the other hand, there were significant differences in the emotional problems and peer relationship problems subscales between the three clusters. The Scheffe test confirmed that cluster 3 had a significantly higher frequency of peer relationship and emotional problems than did clusters 1 and 2. Table 2 shows the strengths and difficulties of the three clusters.

\section{School life of the three clusters}

The differences in school life between the three clusters were analyzed. The three clusters differed in school atten- dance and punishments received from school. The analysis of residual structures using Pearson residuals for a post-hoc test revealed that cluster 1 had a significantly lower percentage of students reported to have had poor attendance due to early leave and absences relative to the other groups, while cluster 3 had a significantly higher percentage of students reported to have poor attendance due to early leave and absences compared to the other groups. Regarding school punishments, the percentage of students who received punishment in school was significantly lower in cluster 1 and significantly higher in cluster 2 than in the other groups. There were no significant differences in the percentage of students deemed to be in need of management after school-based mental health screening test: $10.4 \%$ in cluster $1,12.5 \%$ in cluster 2 , and $24.4 \%$ in cluster 3 . The three clusters differed significantly in school belongingness and relationships with teachers. Scheffe's test confirmed that clusters 1 and 2 had a higher sense of school belongingness than did cluster 3 , with no significant difference between clusters 1 and 2. Cluster 1 had better relationships with teachers than did cluster 3 , with no significant differences between clusters 1 and 2 and between clusters 2 and 3. There were no significant differences between the clusters in terms of the distribution of school grades. Table 3 shows the school life-related characteristics of the three clusters.

Table 1. The demographic characteristics of cluster types

\begin{tabular}{|c|c|c|c|c|c|}
\hline Variable & Cluster $1(n=84)$ & Cluster $2(n=42)$ & Cluster $3(n=47)$ & $\chi^{2} / F$ & Post-hoc (Scheffe) \\
\hline Sex & & & & 5.82 & \\
\hline Male & $54(64.3)$ & $23(54.8)$ & $20(42.6)$ & & \\
\hline Female & $30(35.7)$ & $19(45.2)$ & $27(57.4)$ & & \\
\hline Age (years) & $15.80 \pm 1.80$ & $16.10 \pm 1.81$ & $16.47 \pm 1.57$ & 2.17 & \\
\hline Level of school & & & & 7.15 & \\
\hline High school & $47(56.0)$ & $29(69.0)$ & $36(76.6)$ & & \\
\hline Middle school & $33(39.3)$ & $12(28.6)$ & $11(23.4)$ & & \\
\hline Elementary school & $4(4.8)$ & $1(2.4)$ & $0(0.0)$ & & \\
\hline Socioeconomic status* & & & & $28.68^{\dagger}$ & \\
\hline High & $10(13.2)$ & $2(5.4)$ & $8(18.2)$ & & \\
\hline Middle & $60(78.9)$ & $16(43.2)$ & $24(54.5)$ & & \\
\hline Low & $6(7.9)$ & $19(51.4)$ & $12(27.3)$ & & \\
\hline Intimacy with parents & $4.52 \pm 1.25$ & $3.03 \pm 1.45$ & $3.28 \pm 1.52$ & $20.49^{\dagger}$ & $1>2,3$ \\
\hline
\end{tabular}

Data are presented as $\mathrm{n}(\%)$ or mean \pm standard deviation. ${ }^{*}$ the ratio of missing value is $9.20 \%,{ }^{\dagger} p<0.001$

Table 2. Strengths and difficulties among cluster types

\begin{tabular}{|c|c|c|c|c|c|}
\hline Variables & Cluster $1(n=84)$ & Cluster $2(n=42)$ & Cluster $3(n=47)$ & $\mathrm{F}$ & Post-hoc (Scheffe) \\
\hline SDQ_prosocial behaviour & $6.62(2.12)$ & $6.05(2.49)$ & $5.60(2.46)$ & 3.04 & \\
\hline SDQ_hyperactivity & $2.21(2.11)$ & $2.70(2.14)$ & $2.50(1.81)$ & 0.89 & \\
\hline SDQ_peer problems & $1.56(1.43)$ & $1.42(1.10)$ & $3.31(2.11)$ & $22.27^{*}$ & $3>1,2$ \\
\hline SDQ_emotioanl symptoms & $1.20(1.40)$ & $1.79(1.52)$ & $4.15(2.28)$ & $46.01^{*}$ & $3>1,2$ \\
\hline SDQ_conduct problems & $0.97(0.10)$ & $1.49(1.38)$ & $1.24(1.25)$ & $2.90^{*}$ & \\
\hline
\end{tabular}

Data are presented as mean (standard deviation). The ratio of missing values is $1.16 \%$. ${ }^{*} p<0.05$. SDQ: Strengths and Difficulties Questionnaire 
Table 3. School factors among cluster types

\begin{tabular}{|c|c|c|c|c|c|}
\hline Variables & Cluster $1(n=84)$ & Cluster $2(n=42)$ & Cluster $3(n=47)$ & $\chi^{2} / F$ & Post-hoc (Scheffe) \\
\hline Truancyn & $8(9.5)$ & $15(35.7)$ & $27(58.7)$ & 36.04 & \\
\hline Disciplinary & $2(2.4)$ & $10(23.8)$ & $4(8.5)$ & 15.19 & \\
\hline High-risk group* & $8(10.4)$ & $5(12.5)$ & $10(24.4)$ & 4.40 & \\
\hline Belongingness to school & $3.38 \pm 0.67$ & $3.20 \pm 0.72$ & $2.66 \pm 0.87$ & $14.19^{\ddagger}$ & $1,2>3$ \\
\hline Relationship with teacher & $3.59 \pm 0.61$ & $3.35 \pm 0.53$ & $3.19 \pm 0.71$ & $6.29^{\dagger}$ & $1>3$ \\
\hline
\end{tabular}

Data are presented as $\mathrm{n}(\%)$ or mean \pm standard deviation. Missing values are within the $0.6-8.7 \%$ range. ${ }^{*}$ students who were classified as high-risk group who have mental health problems requiring intervention in nationwide school-based mental health screening test, ${ }^{\dagger} p<0.01,{ }^{\ddagger} p<0.001$

Table 4. Differences in clinical characteristics among cluster types

\begin{tabular}{|c|c|c|c|c|}
\hline Variables & Cluster $1(n=84)$ & Cluster $2(n=42)$ & Cluster $3(n=47)$ & $\chi^{2}$ \\
\hline Non-suicidal self-injury & $1(1.2)$ & $1(2.5)$ & $13(30.2)$ & 31.51 \\
\hline Addiction problems & $10(13.0)$ & $6(16.2)$ & $11(25.0)$ & 0.24 \\
\hline Individual problems & $13(16.9)$ & $14(34.1)$ & $32(71.1)$ & $36.26^{\dagger}$ \\
\hline Learning problems & $33(41.3)$ & $13(32.5)$ & $23(48.9)$ & 2.41 \\
\hline Family problems & $14(22.2)$ & $26(66.7)$ & $25(59.5)$ & $24.27^{\dagger}$ \\
\hline Peer problems & $4(5.1)$ & $9(22.5)$ & $8(18.2)$ & $8.70^{*}$ \\
\hline Incidents just before suicide & $23(39.0)$ & $19(32.2)$ & $17(28.8)$ & 4.61 \\
\hline
\end{tabular}

Data are presented as $n(\%)$. Missing values are within the $3.5-6.0 \%$ range. ${ }^{*} p<0.05,{ }^{\dagger} p<0.001$

Table 5. Warning signs among cluster types

\begin{tabular}{lccccc}
\hline \multicolumn{1}{c}{ Variables } & Cluster $1(\mathrm{n}=80)$ & Cluster 2 $(\mathrm{n}=39)$ & Cluster 3 $(\mathrm{n}=46)$ & $\mathrm{F}$ & Post-hoc $(\mathrm{scheffe})$ \\
\hline Verbal signs & $0.01(0.11)$ & $0.05(0.22)$ & $0.41(0.68)$ & $16.95^{*}$ & $3>1,2$ \\
Behavioral signs & $0.03(0.16)$ & $0.15(0.43)$ & $0.60(1.02)$ & $14.70^{*}$ & $3>1,2$ \\
Emotional signs & $0.03(0.16)$ & $0.08(0.27)$ & $0.36(0.60)$ & $13.72^{*}$ & $3>1,2$ \\
Total scores & $0.06(0.29)$ & $0.28(0.69)$ & $1.39(2.04)$ & $20.55^{*}$ & $3>1,2$ \\
\hline
\end{tabular}

Data are presented as mean (standard deviation). The ratio of missing values is $4.6 \% .{ }^{*} p<0.001$

\section{Circumstances preceding the time of death of the three clusters}

We performed Pearson's chi-squared test to assess the presentation of problems in the following five areas in the year preceding suicide: personal, addiction, family, friend, and school studies. The results showed that the three clusters differed significantly in personal, family, and friend problems but not in problems related to addiction or studies. The analysis of residual structures using Pearson residuals revealed that cluster 2 and cluster 3 had significantly higher rate of personal and friend problems than cluster 1, while cluster 2 had a higher rate of family problems than did the other clusters. Further, the results of the cluster analysis of the presence of non-suicidal self-injuries were significant. More specifically, cluster 3 had a significantly higher frequency of selfinjury (30.2\%) than did clusters 1 and 2.

The results of the cluster analysis of the presence of evident stressful situations immediately before suicide were not significant. More specifically, $39.0 \%$ of cluster $1,32.2 \%$ of cluster 2 , and $28.8 \%$ of cluster 3 had experienced an evident stressful situation in the year preceding the suicide. The most common stressful event in the entire sample was conflict with parents (19.2\%), followed by others (5.8\%), problem behaviors (3.6\%), and career- and grade-related problems (3.0\%). For a more detailed analysis, we performed a cluster analysis for each of the stressful events (conflict with parents, conflict with friends, dating and relationship problems, conflict with siblings, career- and grade-related events, and problem behaviors). The results confirmed that there were significant differences between the three clusters in dating, relationship problems, and school punishments. Although the rate itself was low, cluster 2 had a significantly higher frequency of dating and relationship problems and problem behaviors than did the other clusters. These results are shown in Table 4.

Regarding pre-suicide warning signs, the total scores for the verbal, behavioral, and emotional sign subscales were computed and compared between the three clusters. Table 5 shows the results. The most evident warning sign in the entire student pool was changes in emotional state, changes in sleep, and self-depreciative words (5.2\% of the sample). The three clusters differed significantly in all three signs. Scheffe's post-hoc test confirmed that cluster 3 showed higher verbal, 
behavioral, and emotional signals than did clusters 1 and 2 . There were no significant differences between clusters 1 and 2 .

\section{DISCUSSION}

This study explored how student suicides can be clustered into the following three types based on six known risk factors for suicide: the silent type, which does not present any of the risk factors; the environmental-risk type, in which broken families and deviant behaviors such as smoking/drinking and running away from home are more common; and the depressive type, which is characterized by depression and the diagnosis of a mental disorder.

The first type of students lacked all of the risk factors of suicide as viewed by their teachers, and they were thus named the silent type. There were twice as many male students than female students in this group, and they had relatively good socioeconomic statuses, strong sense of high school belongingness, good relationships with their teachers, and had adapted to school life well. Furthermore, they had no prior history of mental disorders, suicide attempts, or non-suicidal self-injury, and they were not involved in any stressful events during the period preceding the suicide. Unfortunately, this type of student accounted for $48.6 \%$ of the entire sample. Previous studies have also mentioned a subtype of adolescents who were not predicted to commit suicide [12,18-20]. A British study on the suicides of adolescents reported that adolescents who were relatively well functioning and lacked a clear history of mental disorder or previous suicide attempts comprised $19 \%$ of their sample [19]. They mentioned that students in this group were characterized by unstable personalities, high expectations for themselves, aspirations towards perfection, and tendencies to become overwhelmed by perceived failures. An Israeli school-based psychological autopsy study on adolescents who committed suicide described these adolescents as the "tragic narrative" type, and reported that they accounted for about $20 \%$ of suicide cases [12]. This group of adolescents was reported to experience extreme stress, have difficulties in adaptation, refrain from seeking outside help, and consider seeking help as a personal failure [12]. The percentage of the silent type in our sample was two-fold higher than those reported by the aforementioned studies $[12,19]$. This finding underscores the difficulty of detecting mental health problems in schools and performing timely interventions to prevent suicide in this population. According to a previous study, the vast majority of adolescents at risk of suicide do not seek help due to concerns about confidentiality and potential stigmatization [21]. Considering the high number of students categorized into the silent type, as well as the traits exhibited by this type, school-based supportive interventions need to be provided to seemingly normal students without clear risk factors, and school-based suicide prevention programs should include stress and emotional management, awareness of mental health problems, and reduction of stigmatization for seeking help. On one hand, it is possible that important life events that these students experienced or risk factors, such as previous suicide attempts, may not have been evident to their teachers [22]. Therefore, gatekeeper training should be provided to school staff and faculty to equip them to detect minor changes in students.

The second type accounted for $24.28 \%$ of the entire sample. These students were of a relatively low socioeconomic status compared to other types, more than $80 \%$ of the students were members of broken families, and about one-third of the students demonstrated deviant behaviors. This group was named the environmental-risk type. About $23 \%$ of these students had received punishment at school, and a substantial number of the students experienced family conflicts. Further, some students experienced problems due to their behaviors having been exposed or had dating and relationship problems around the time of their suicide. On the other hand, these students were not conspicuously depressed or anxious as observed by their teachers, had not received diagnoses for any mental disorders, and had no prior self-injury or suicide attempts. Past studies have reported that family breakup caused by divorce, bereavement, or separation are clear risk factors for suicide $[4,5]$. A Korean study also reported family breakup to be a significant risk factor for suicidal behaviors among adolescents [23]. Our study results suggest that the loss of a family member is likely to exacerbate deviant behaviors and thus expose the children to an elevated risk of suicide. Deviant behaviors are associated with aggression and impulsivity [24], and are key risk factors for suicide [25]. Considering that the depressive symptoms of the children in this group passed unobserved by teachers, it is possible that these students would not be identified by the screening of suicide risk based on depressive symptoms. In the future, closer attention should be paid to suicidal behaviors and deviant behaviors in adolescents who lost a family member, and interventions that induce psychological stress caused by family environments should be comprehensively considered in suicide prevention interventions for these students.

The third cluster accounted for $27.17 \%$ of the entire sample. Named the depressive type, this group of students was mostly comprised of adolescents who were diagnosed with a mental disorder, complained of depression, and had previously attempted suicide or committed non-suicidal self-injury prior to their suicides. These students not only frequently showed attendance problems, such as unauthorized school absences, but also had a low sense of school belonging and had poor 
relationships with their teachers, thereby exhibiting markedly reduced functioning in school than did the other types of students. Furthermore, they were more likely to show clear risk signs, such as mentioning suicide or showing behavioral changes, immediately before suicide than were the other types of students. While their prior suicide attempts and non-suicidal self-injury were likely a means of expressing their pain, these behaviors seem to have strengthened their suicidal potential [26], and their failure to receive help afterwards seems to have led to suicide. The fact that they had already been diagnosed with a psychiatric disorder and that they clearly showed risk behaviors such as self-injury and suicide attempts suggests that they were the type of students whose suicidal risk would have been most likely detectable. However, the rate at which the risk signs were observed by the teachers was found to be very low in this study. This contrasts with the results of a Korean psychological autopsy study, which found that $92.3 \%$ of the individuals who died from suicide exhibited warning signs within three months of their death [27]. This difference may be attributable to the person reporting the warning signs: i.e., whether the teachers or family members submitted the report. Psychological autopsy reports on family members are the result of a close analysis of lives of deceased and other records, but the teacher's school suicide reports have practical limitations in identifying the status of suicide students in more detail. Despite the high rate of risk factors among the third type of students, our study found that warning signs were not effectively detected around the time of death, indicating the need for increased awareness of the warning signals exhibited by adolescents at high risk of suicide by the school staff and faculty, as well as families. In addition, treatment targeting suicidal risk and intensive crisis interventions are also needed.

The results of this study suggest that family breakup and mental disorders, which have been identified in association with suicide by past studies [3,4], can independently increase the risk of suicide as reflected by deviant behaviors and selfmutilation/suicide attempts, respectively. Furthermore, we observed a silent type of students who committed suicide; students in this group did not express known risk factors for suicide, and any changes in their behavior were not detected by their teachers. However, considering that the rate of mental disorder was relatively low in the entire sample, we also speculate that the family-risk type and silent-type students had mental disorders that had not been diagnosed or treated at the times of their death. Further, considering that many people in Korea with mental disorders do not seek aggressive treatment because they either fear stigmatization or downplay the gravity of mental health problems, there is a need for continuous effort to improve the prioritization of mental health and reduce the stigma associated with mental disease.

This study is subject to a few limitations. First, data from 75 adolescents were excluded from the analysis due to missing values for major variables used in cluster analysis. The consequently reduced sample size limits the generalizability of our findings. This limitation further indicates the possibility that our study data, which were reported by teachers, may not have appropriately reflected the subjects' situations and traits. Subsequent studies should integrate data from various sources, including family reports, and conduct in-depth interviews. Second, the subjects were clustered based only on six risk factors-despite a variety of other candidate risk factors such as exposure to childhood trauma or a family history of suicide. Nevertheless, this study is significant in that it detected various risk factors and pre-suicide signs among adolescents who committed suicide based on teachers' reports and clustered them into different types. Another limitation of this study was that we performed the cluster analysis of the risk factors at the same time point, although the temporal distance from these risk factors to suicide death might have differed. Nevertheless, we presented the process through which adolescents progress to suicide and suggest that different warning signs may be considered in establishing suicide prevention interventions strategies tailored to specific possible trajectories.

\section{CONCLUSION}

This study explored how student suicides can be clustered based on the six known risk factors for suicide. Cluster analysis identified three categories: the silent type, which does not present any risk factors; the environmental-risk type, in broken families and deviant behaviors such as smoking/drinking, and running away from home are more frequent; and the depressive type, which is characterized by depression and a history of mental disorder. In the future, continuous and longterm studies are needed to identify further risk factors of suicide among adolescents.

\section{Acknowledgments}

This work was supported by the Ministry of Education of the Republic of Korea and the National Research Foundation of Korea (NRF-2018S1A5B8A02081988).

\section{Conflicts of Interest}

The authors have no potential conflicts of interest to disclose.

\section{Author Contributions}

Resources: Hyun Ju Hong. Supervision: Yong-Sil Kweon. Writitingoriginal draft: Hoin Kwon. Writing—review \& editing: Yong-Sil Kweon, Hyun Ju Hong.

\section{ORCID iDs}

Hoin Kwon https://orcid.org/0000-0001-6323-6908 
Hyun Ju Hong ～https://orcid.org/0000-0002-6348-9996

Yong-Sil Kweon https://orcid.org/0000-0001-5638-6350

\section{REFERENCES}

1) World Health Organization. Suicide [cited $2020 \mathrm{Sep} 2$ ]. Available from URL: https://www.who.int/news-room/fact-sheets/detail/suicide.

2) Statistics Korea. Annual report on the cause of death statistics 2019 [cited $2020 \mathrm{Jul}$ 2]. Available from URL: http://kostat.go.kr/ portal $/$ korea/kor_nw/1/6/2/index.board?bmode $=$ read\&bSeq $=\& a S$ $\mathrm{eq}=385219 \&$ pageNo $=1 \&$ rowNum $=10 \&$ navCount $=10 \&$ currPg $=\& \mathrm{~s}$ earchInfo $=\&$ sTarget $=$ title \&sTxt $=$.

3) Mann JJ, Apter A, Bertolote J, Beautrais A, Currier D, Haas A, et al. Suicide prevention strategies: a systematic review. JAMA 2005; 294:2064-2074.

4) Brent DA, Perper JA, Moritz G, Allman C, Friend A, Roth C, et al. Psychiatric risk factors for adolescent suicide: a case-control study. J Am Acad Child Adolesc Psychiatry 1993;32:521-529.

5) Gould MS, Fisher P, Parides M, Flory M, Shaffer D. Psychosocial risk factors of child and adolescent completed suicide. Arch Gen Psychiatry 1996;53:1155-1162.

6) Brent DA, Johnson BA, Perper J, Connolly J, Bridge J, Bartle S, et al. Personality disorder, personality traits, impulsive violence, and completed suicide in adolescents. J Am Acad Child Adolesc Psychiatry 1994;33:1080-1086.

7) Nock MK, Green JG, Hwang I, McLaughlin KA, Sampson NA, Zaslavsky AM, et al. Prevalence, correlates, and treatment of lifetime suicidal behavior among adolescents: results from the National Comorbidity Survey Replication Adolescent Supplement. JAMA Psychiatry 2013;70:300-310.

8) Bentley KH, Franklin JC, Ribeiro JD, Kleiman EM, Fox KR, Nock MK. Anxiety and its disorders as risk factors for suicidal thoughts and behaviors: a meta-analytic review. Clin Psychol Rev 2016;43: 30-46.

9) Allebeck P, Allgulander C. Suicide among young men: psychiatric illness, deviant behaviour and substance abuse. Acta Psychiatr Scand 1990;81:565-570.

10) Jae YM. Suicide and psychiatric disorder. J Korean Soc Biol Ther Psychiatry 2004;10:3-10.

11) Lazear K, Roggenbaum S, Blase K. Youth suicide prevention schoolbased guide. Tampa, FL: Department of Child and Family Studies, Division of State and Local Support, Louis de la Parte Florida Mental Health Institute, University of South Florida;2003.

12) Zalsman G, Siman Tov Y, Tzuriel D, Shoval G, Barzilay R, Tiech Fire N, et al. Psychological autopsy of seventy high school suicides: combined qualitative/quantitative approach. Eur Psychiatry
2016;38:8-14.

13) Suicide and School Mental Health Institutes. 2015 School based psychological autopsy. Chuncheon: Suicide and School Mental Health Institutes;2016.

14) Suicide and School Mental Health Institutes. 2018 Student suicide death and attempt issue reports. Chuncheon: Suicide and School Mental Health Institutes;2019.

15) Suicide and School Mental Health Institutes. 2019 Student suicide death Report. Chuncheon: Suicide and School Mental Health Institutes; 2020

16) Ahn JS, Jun SK, Han JK, Noh KS, Goodman R. The development of a Korean version of the strengths and difficulties questionnaires. J Korean Neuropsychiatr Assoc 2003;42:141-147.

17) Kaufman L, Rousseeuw PJ. Finding groups in data: an introduction to cluster analysis. Hoboken, NJ: John Wiley \& Sons, Inc.; 1990.

18) Beautrais AL. Suicides and serious suicide attempts: two populations or one? Psychol Med 2001;31:837-845.

19) Fortune S, Stewart A, Yadav V, Hawton K. Suicide in adolescents: using life charts to understand the suicidal process. J Affect Disord 2007;100:199-210.

20) Portzky G, Audenaert K, van Heeringen K. Suicide among adolescents. A psychological autopsy study of psychiatric, psychosocial and personality-related risk factors. Soc Psychiatry Psychiatr Epidemiol 2005;40:922-930.

21) Hawton K, Rodham K, Evans E, Weatherall R. Deliberate self harm in adolescents: self report survey in schools in England. BMJ 2002;325:1207-1211.

22) Hawton K, Saunders KE, O'Connor RC. Self-harm and suicide in adolescents. Lancet 2012;379:2373-2382.

23) Kwon H, Lee JS, Kim AR, Hong HJ, Kweon YS. Risk factors for suicidal ideation and attempts in adolescents. J Korean Acad Child Adolesc Psychiatry 2018;29:114-121.

24) Bukstein OG, Brent DA, Perper JA, Moritz G, Baugher M, Schweers J, et al. Risk factors for completed suicide among adolescents with a lifetime history of substance abuse: a case-control study. Acta Psychiatr Scand 1993;88:403-408.

25) Renaud J, Berlim MT, McGirr A, Tousignant M, Turecki G. Current psychiatric morbidity, aggression/impulsivity, and personality dimensions in child and adolescent suicide: a case-control study. J Affect Disord 2008;105:221-228.

26) Joiner TE, Van Orden KA. The interpersonal psychological theory of suicidal behavior indicates specific and crucial psychotherapeutic targets. Int J Cogn Ther 2008;1:80-89.

27) Korea psychological autopsy center. Psychological interview report [cited $2020 \mathrm{Jul}$ 2]. Available from URL: http://www.psyauto.or.kr/ sub/data_02.asp. 